\title{
Multiple Cranial Neuropathies Caused by Lymphoma-Associated Central Nervous System Involvement in an Immunodeficiency Virus Patient
}

Sumonthip Leelawai, M.D., Pat Korathanakhun, M.D.

Division of Neurology, Department of Internal Medicine, Faculty of Medicine, Prince of Songkla University, Hat Yai, Songkhla 90110, Thailand.

Received 20 September 2019 • Revised 13 November 2019 • Accepted 23 November 2019 • Published online 26 December 2019

\begin{abstract}
:
Human immunodeficiency virus (HIV) infections increase the risk of malignant lymphoma. Although, lymphoma is a rare cause of central nervous system involvement, it can present as an initial HIV symptom. We report the case of a 35-year-old HIV patient who developed multiple cranial neuropathies. He was diagnosed with diffuse large B-cell lymphoma with meningeal metastasis.
\end{abstract}

Keywords: cranial neuropathies, HIV, lymphoma

\section{Introduction}

Human immunodeficiency virus (HIV) infection is associated with a significantly increased risk for malignant lymphoma. ${ }^{1}$ Extranodal involvement, in particular at the meninges and brain metastasis, was reported to occur in $20.0 \%$ of non-Hodgkin lymphoma (NHL) patients, and is related to systemic acquired immune deficiency syndrome (AIDS). ${ }^{2}$ The involvement of lymphoma in the central nervous systems (CNS) can frequently present as an initial symptom of HIV infection. ${ }^{3}$ We report a case of lymphoma in an HIV patient who presented with multiple cranial neuropathies due to meningeal metastasis.
Contact: Pat Korathanakhun, M.D.

Division of Neurology, Department of Internal Medicine, Faculty of Medicine, Prince of Songkla University, Hat Yai, Songkhla 90110, Thailand.

E-mail: patosk120@gmail.com

This is an open access article under the CC BY-NC-ND license

(http://www.jhsmr.org/index.php/jhsmr/about/editorialPolicies\#openAccessPolicy). 


\section{Case report}

A 35-year-old Thai male was admitted to the hospital due to left hemiparesis and diplopia for 1 week. One month prior to admission, he had developed subacute progressive low back pain which radiated to the left leg. His pain was relieved with pain medication. He also had allodynia in the left thumb and index finger at that time, without any weakness or numbness. About two weeks after the appearance of these initial symptoms he had a gradual onset of persistent, dull aching epigastrium pain without radiation, nausea, vomiting, dysphagia, diarrhea, or jaundice. One week prior to admission, he developed left facial palsy with incomplete left eye closure and the inability to raise his left eyebrow. He also had weakness in his left arm and leg. A few days later, he developed right facial weakness and horizontal diplopia, which was worst in the right lateral gaze; however, there was no facial numbness. He also suffered from anorexia and weight loss, but there were no systemic symptoms suggestive of other organ involvement at that time. These problems finally brought him to the hospital, where upon admission a general physical examination noted a cachexic appearance, and mild tenderness at the epigastrium. There was no superficial lymphadenopathy, and the liver span was 14 centimeters $(\mathrm{cm})$ at the midclavicular line.
The neurological examination revealed abduction deficit of the right eye, which suggested right abducens nerve palsy (Figure 1). He had no ptosis and the pupils were equal in size and both eyes reacted to light. He had bilateral lower motor neuron type facial palsy. Examination of the other cranial nerves was within normal limits. A motor system examination revealed no muscle atrophy, normal muscle tone and motor power on the right upper and lower extremities; however, there was flaccid muscle tone and motor power of grade $\|$ at the left upper and lower extremities.

Pinprick sensation impairment was found at the left C6-C7 dermatomes. The proprioceptive sensation test was normal. Deep tendon reflexes were hyporeactive in all extremities. The other neurological signs and general systemic signs were normal.

The complete blood count showed leukopenia (white blood cell count (WBC) 3.65 $\times 10^{3} /$ microliters, neutrophils $75.0 \%$, lymphocytes $13.0 \%$ ) with young WBCs (myelocytes $3.0 \%$, metamyelocytes $3.0 \%$ ), mild anemia (hemoglobin $10.8 \mathrm{gram} /$ deciliter) and normal platelet count $\left(191 \times 10^{3} /\right.$ microliter). The liver function test values showed mildly elevated aspartate aminotransferase (AST) and alanine aminotransferase (ALT) [AST 111 units per liter (U/), ALT $39 \cup /$ ]. Serum lactate dehydro-
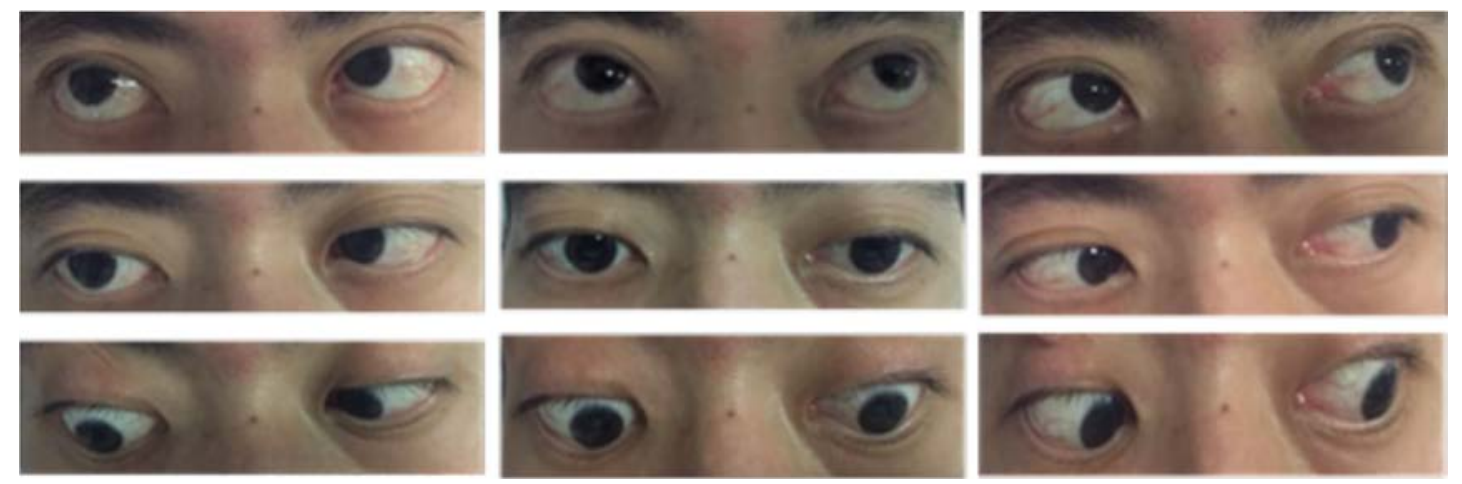

Figure 1 Extraocular muscle examination showing limited abduction of the right eye, suggesting right abducens nerve palsy. The other extraocular muscle movements were within normal limits. 
genase was also elevated (7201 U/L). The anti-HIV antibody test was positive, with clusters of differentiation 4 T-lymphocyte; absolute count of 22 cells/cubic millimeter $\left(\mathrm{mm}^{3}\right)$ (CD4+ T-lymphocytes $6.0 \%$ of total lymphocytes). Hepatitis B, C virus profiles, venereal disease research laboratory (VDRL) and cryptococcal antigen tests were negative.

Magnetic resonance imaging (MRI) of the brain showed gadolinium enhancement of bilateral cranial nerve III, internal acoustic canals, right cranial nerve $\mathrm{X}$, and cranial nerve XI (Figure 2). MRI of the cervical and thoracolumbar spine showed heterogeneous signal intensity and gadolinium enhancement of the spinal vertebral marrow with homogeneous enhancement of anterior epidural soft tissue lesions at L2 lumbar spine and upper sacrum (Figure 3 ). The neuroradiological findings were compatible with lymphomatous infiltration. MRI of the upper abdomen showed two liver masses (3.6×3.7 and $3.0 \times 4.2 \mathrm{~cm}$ ) with signal hypointensity on T1 weighted image, hyperintensity on T2 weighted image, and restrictive diffusion-weighted imaging.
Cerebrospinal fluid (CSF) analysis revealed minimally elevated WBC (WBC 9 cells $/ \mathrm{mm}^{3}$, mononuclear cells $7.0 \%$, polymorphonuclear cells $9.0 \%$ ) and high CSF protein [341 milligram\% (mg\%)]. The CSF glucose was 18 $\mathrm{mg} \%$, and serum sugar was $50 \mathrm{mg} \%$. The CSF cytology was negative for malignancy.

The pathological findings of a liver biopsy reported high-grade B-cell neoplasm, which was consistent with diffuse large B-cell lymphoma (DLBCL) (Figure 4). Bone marrow aspiration and biopsy revealed extensive DLBCL involvement (Figure 5).

During hishospital stay, hedeveloped severe lactic acidosis with hypoglycemia, which was compatible with the Warburg effect. Although, the metabolic acidosis was reversed by peritoneal dialysis, the functional status of the patient was not suitable for chemotherapy, thus a high dose of steroids was administered instead to reduce the number of tumor cells. Shortly after this the patient developed severe pneumonia, and subsequently died of acute respiratory failure and septic shock.
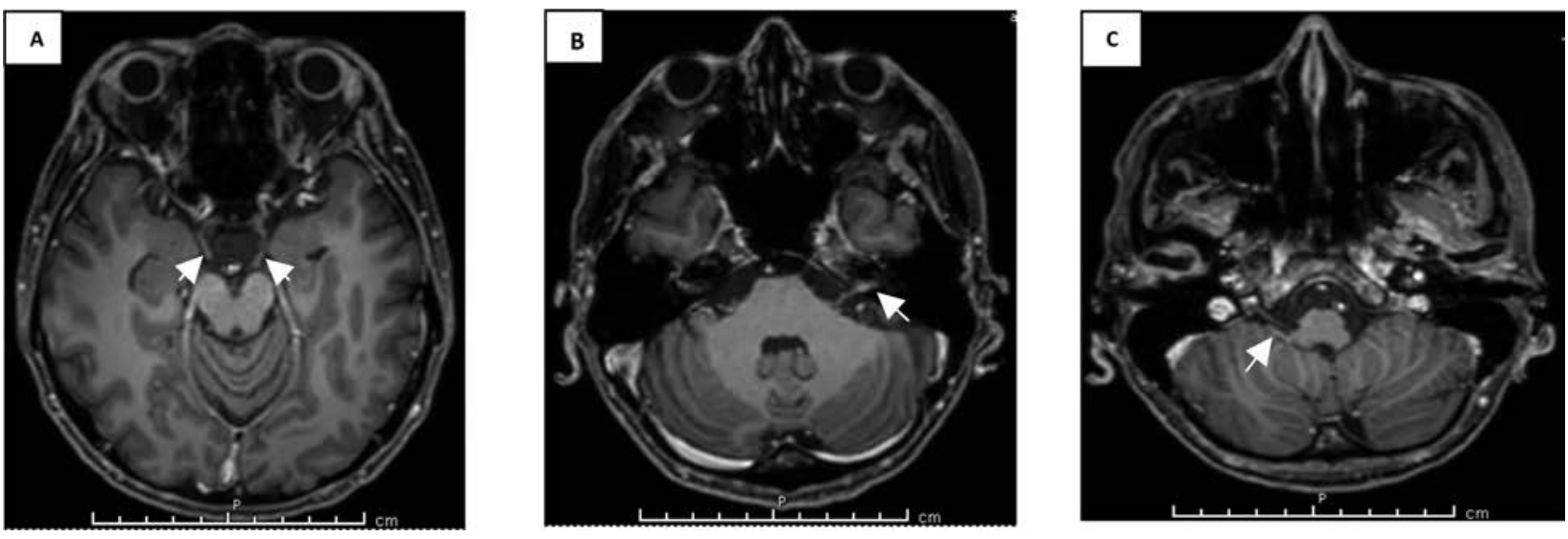

Figure 2 T1-weighted MRI of the brain in T1, with gadolinium showing gadolinium enhancement of the bilateral cranial nerve III (A), internal acoustic canals $(B)$, right cranial nerve $X$ and $X I(C)$ 


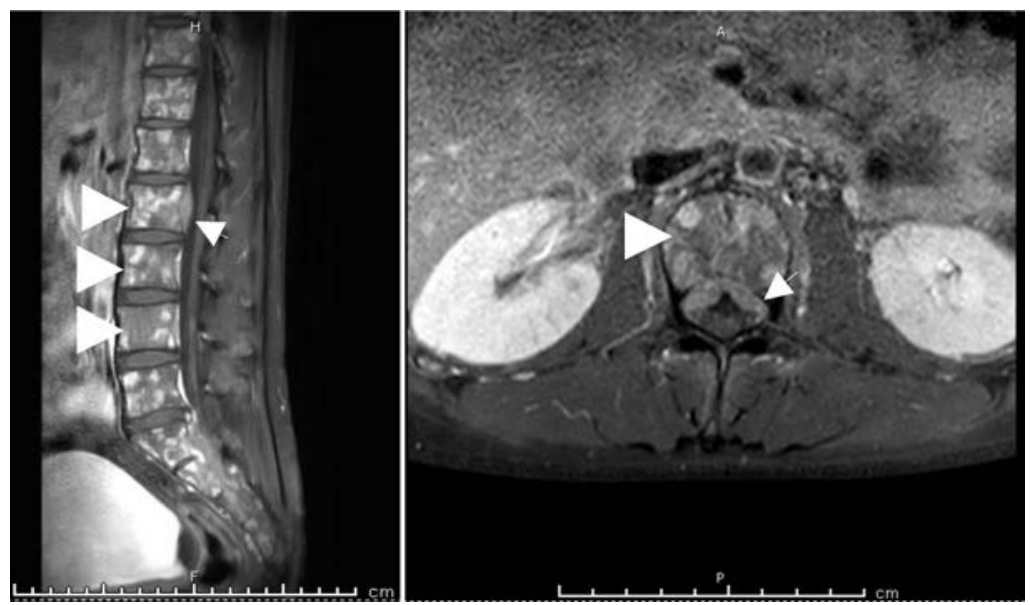

Figure $3 \mathrm{~T} 1$-weighted $\mathrm{MRI}$ of the thoracolumbar spine with gadolinium showing heterogeneous signal intensity, and gadolinium enhancement of the spinal vertebral marrow with homogeneous enhancement of anterior epidural soft tissue lesions at L2 lumbar spine and upper sacrum.

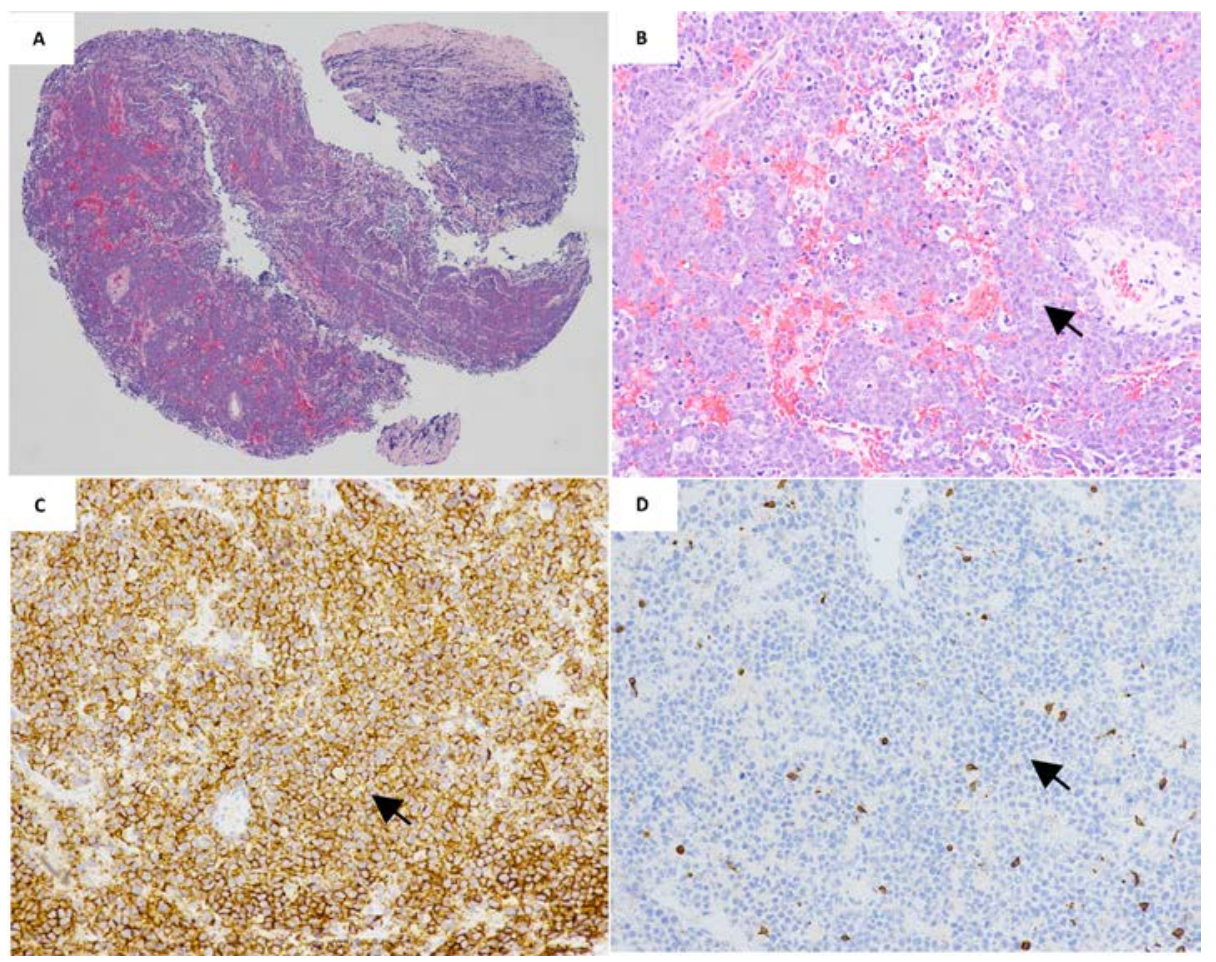

Figure 4 Liver biopsy results showing infiltration of a high grade B-cell neoplasm, consistent with diffuse large B-cell lymphoma. (A - 4x H\&E, B - 20x H\&E) Immunostaining demonstrated tumor cells marked with CD20, but not marked with CD3. (C - 20x CD20, D - 20x CD3) 


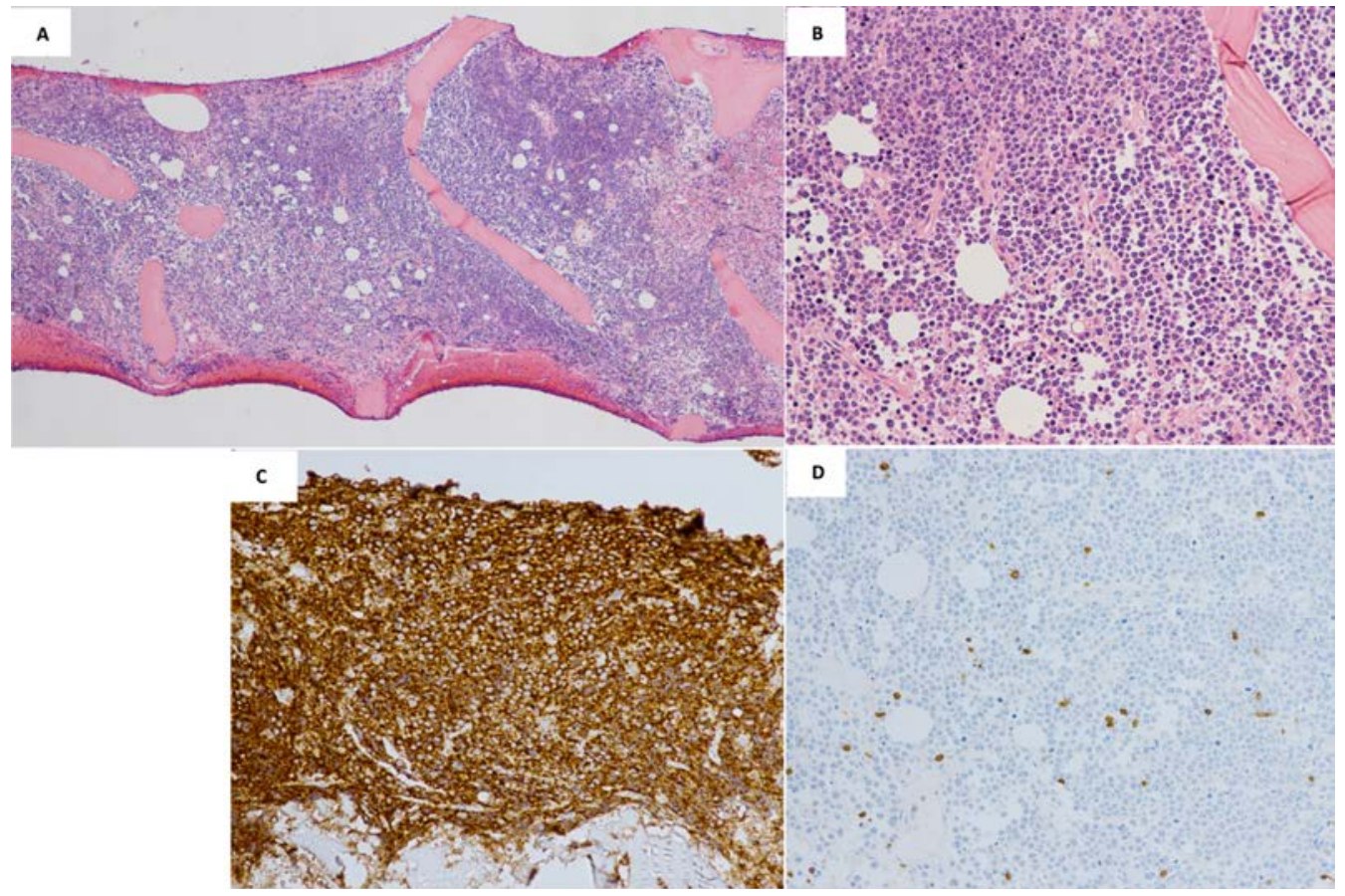

Figure 5 Bone marrow biopsy shows extensive involvement of a large diffuse I B-cell lymphoma. The tumor involves about $98.0 \%$ of the bone marrow. ( $\mathrm{A}-4 \mathrm{x} \mathrm{H} \& \mathrm{E}, \mathrm{B}-20 \mathrm{x} \mathrm{H} \& \mathrm{E})$ Immunostaining demonstrated that the tumor cells were marked with CD20, but not marked with CD3. (C - 20x CD20, D - 20x CD3)

\section{Discussion}

This case report describes CNS involvement of $\mathrm{NHL}$ as an initial presentation in an HIV patient. Based on the current classification by World Health Organization (2016), the subtypes of NHL include mature B-cell neoplasms, mature T-cell and natural killer-cell neoplasms. ${ }^{4}$ A meta-analysis that included data on more than 400,000 patients from seven cancer registries demonstrated a 23-fold increased risk of $\mathrm{NHL}$ development in the HIV-positive population relative to the general population. ${ }^{5}$ Burkitt lymphoma, and DLBCL are considered as the most common subtypes of lymphomas in HIV patients. ${ }^{6}$

Regarding the systemic involvement of $\mathrm{NHL}$, one study reported that extranodal involvement was significantly involved more frequently in HIV-positive patients than in HIV-negative patients. ${ }^{3}$ Other studies, focusing on
HIV groups, found that, over half of the extranodal NHLs involved the upper aerodigestive and gastrointestinal tracts. ${ }^{7,8}$ Another study reported that, although CNS involvement was found in fewer cases, the NHL preferentially affected the meninges; in the form of tumor infiltration surrounding both facial and cranial nerves supplying the extraocular muscles. $^{9}$

Two studies reported that, the most common sites of extranodal involvement of NHLs in HIV patients were bone marrow (26.0\%), liver (20.5\%), kidney (7.7\%) and bone $(7.7 \%) .{ }^{7,8}$ However, CNS involvement of $\mathrm{NHL}$ is uncommon in Thailand. The most common pattern of metastatic involvement of the nervous system is metastasis to the subarachnoid space, also referred to as the leptomeningeal spread. ${ }^{1}$ 
$\mathrm{NHL}$ is one of the three most common AIDS-defining cancers, the other two being; Kaposi sarcoma and cervical cancer. The pathogenesis of lymphoma in HIV infection is related to the transforming properties of the retrovirus itself, the immunosuppression and cytokine dysregulation that result from the disease, and opportunistic infections. Ever since highly active antiretroviral therapy (HAART) have become available, the rates of $\mathrm{NHL}$ have declined by $30.0-50.0 \%$ in HIV patients, compared to before the HAART era. HAART allows for prolonged suppression of HIV replication and an improved immune status, as manifested in rising CD4 counts. ${ }^{1}$

\section{Conclusion}

The incidence of lymphoma in patients with HIV infection greatly exceeds that of the general population. Although, CNS involvement is a rare and unexpected complication of NHL, it can present as the initial symptom. Therefore, awareness of the possible existence of extranodal lymphoma should lead to a rapid and correct diagnosis.

\section{Acknowledgement}

We highly appreciate Nuttha Sunghan, M.D. for the assistance in the neuroimaging review and Kanet Kanjanapradit, M.D. for pathological review.

\section{Funding sources}

This manuscript was funded by the Faculty of Medicine, Prince of Songkla University, Thailand.

\section{Conflict of interest}

The authors declare no conflicts of interest.

\section{References}

1. Grogg KL, Miller RF, Dogan A. HIV infection and lymphoma. J Clin Pathol 2007;60:1365-72.

2. Bomgaars L, Chamberlain MC, Poplack DG, Blaney S. Leptomeningeal metastases. In: Levin VA, editor. Cancer in the nervous system. $2^{\text {nd }}$ ed. New York: Oxford University Press; 2002;p.375-93.

3. Powles T, Matthews G, Bower M. AIDS related systemic nonHodgkin's lymphoma. Sex Transm Infect 2000;76:335-41.

4. Swerdlow SH, Campo E, Pileri SA, Harris NL, Stein H, Siebert R, et al. The 2016 revision of the World Health Organization classification of lymphoid neoplasms. Blood 2016; 127:2375-90.

5. Grulich $\mathrm{AE}$, van Leeuwen MT, Falster MO, Vajdic CM. Incidence of cancers in people with HIV/AIDS compared with immunosuppressed transplant recipients: a meta-analysis. Lancet 2007;370:59-67.

6. Gloghini A, Carbone A, Pantanowitz L. HIV-associated lymphomas. Atlas Genet Cytogenet Oncol Haematol 2017;21: 25-9.

7. Sukpanichnant S, Sonakul D, Piankijagum A, Wanachiwanawin W, Veerakul G, Mahasandana C, et al. Malignant lymphoma in Thailand: changes in the frequency of malignant lymphoma determined from a histopathologic and immunophenotypic analysis of 425 cases at Siriraj Hospital. Cancer 1998;83:1197-204.

8. Makruasi N, Niparuck P, Sungkanuparph S, Ungkanont A, Kanoksil W, Boonsakan P, et al. Non Hodgkin's Lymphoma (NHL) in HIV infected patients in Thailand. J Infect Dis Antimicrob Agents 2011;28:5-14.

9. Katyal N, Wadhwa A, Bollu PC. Multiple cranial neuropathies in a patient with diffuse large B-cell lymphoma: case report and review of literature. Cureus 2018;10:e2186. 